\title{
Evaluation of the effect of the WOX family genes overexpression on the somatic embryogenesis
}

Yakovleva D.V.*, Tvorogova V.E., Lutova L.A.

St. Petersburg State University, St. Petersburg, Russia

*email:dawarus@yandex.ru

Many plant species have an ability to regenerate through somatic embryogenesis (SE) the process of non-zygotic cells transformation into embryogenic cells, which are able to form a new plant going through characteristic stages of embryonic development. SE is widely used in plant biotechnology as a method of plant reproduction in vitro and it has its applications in plant transformation, breeding, artificial seeds production, but the genetic regulation of SE has not yet been clearly understood. The identification of the regulators of this process is important for improving the methods of obtaining somatic embryos.

Previously in our studies, it was found that overexpression of MtWOX9-1 and STENOFOLIA genes from the WUSCHEL-RELATED HOMEOBOX family of transcription factors leads to an increase in the capacity for SE in Medicago truncatula. We hypothesized that other WOX genes of M. truncatula can also have an impact on the SE.

The aim of this study is to search for new WOX genes, which can be involved in SE regulation in $M$. truncatula. We found that several $M$. truncatula genes from the WOX family increase their expression level during SE or in the generative organs. We suppose that the change in the function of these genes could have an effect on the SE capacity. We are planning to obtain modified plants with overexpression of these WOX genes and to measure the ability of such plants to form somatic embryos in vitro. At this moment, several vector constructions for overexpression are obtained and we are going to introduce them into $M$. truncatula plants. If there are interesting cases of WOX genes overexpression manifestations, CRISPR/Cas9 system will be used to inactivate such WOX genes and to explore further their functions during SE.

Acknowledgements: The research was supported by the RFBR (20-016-00124). 